The following paper posted here is not the official IEEE published version. The final published version of this paper can be found in the Proceedings of the IEEE International Conference on Geoscience and Remote Sensing Symposium (2006 : Denver, Colorado):pp. 4040-4043

Copyright @ 2006 IEEE.

Personal use of this material is permitted. However, permission to reprint/republish this material for advertising or promotional purposes or for creating new collective works for resale or redistribution to servers or lists, or to reuse any copyrighted component of this work in other works must be obtained from the IEEE. 


\section{Application of Polarization Coherence Tomography to GB-POLInSAR Data}

\author{
Zheng-Shu Zhou \\ School of Electrical \& Electronic Engineering \\ The University of Adelaide \\ Adelaide, Australia \\ zszhou@eleceng.adelaide.edu.au
}

\author{
Shane R. Cloude \\ AEL Consultants \\ Cupar, Scotland, UK \\ scloude@ieee.org
}

\begin{abstract}
Recently, a new radar imaging technique-Polarization Coherence Tomography or PCT - has been proposed in the literature. This technique uses the variation of interferometric coherence with polarization to reconstruct a vertical profile function in penetrable volume scattering. In this paper we apply PCT for the first time to ground-based polarimetric interferometric data. We first demonstrate how a vertical profile function can be efficiently represented as a Fourier-Legendre series and then show how to reconstruct this series up to second order with only single baseline data. Polarimetric tomographic reconstructions of Australian native plants show the consistencies and merits of PCT compared with other coherence based parameter retrieval models of the polarimetric SAR interferometry technique.
\end{abstract}

Keywords-POLInSAR; ground-based SAR; polarization coherence tomography $(P C T)$

\section{INTRODUCTION}

Polarimetric Synthetic Aperture Radar Interferometry (POLInSAR) is a new radar imaging technology [1] with important application in the remote sensing of vegetation properties such as forest height and biomass as well as emerging applications in agriculture, snow/ice thickness monitoring and urban height and structure applications [2]. Traditionally only simple two layer random media scattering models have been used to estimate a small number of layer parameters from POLInSAR data, such as vegetation height and underlying surface topography. POLInSAR involves generation of interferograms for different transmit/receive polarization pairs [1]. It is then possible to use the change of interferometric phase with polarization information to extract important bio-geophysical parameters [2]. This is especially important in the case of remote sensing of vegetated land surfaces, where polarimetry alone suffers from the inherent height entropy problem while standard single channel interferometry remains underdetermined [3]. Several authors have proposed the processing of multi-baseline data to reconstruct vertical profiles using a range of signal processing methods [3-6], often based on high resolution spectral analysis techniques [4]. On the other hand, Polarization Coherence Tomography (PCT) uses the variation of interferometric coherence with polarization to estimate a vertical structure function [7].

This work was partially supported by the Defence Science and Technology Organisation (DSTO), Australia.
In this paper we attempt to demonstrate the possibility of performing functional reconstruction or tomography using only a single baseline ground-based polarimetric interferometer. In Section II we review the polarimetric coherence tomography algorithm by first summarising a new POLInSAR algorithm to estimate the two important parameters required for PCT, namely height and surface topography and then by reformulating the tomographic reconstruction problem as a Fourier-Legendre series. In Section III we apply this approach to single baseline ground-based polarimetric interferometer data to reconstruct vertical tomograms based on estimation of the coefficients of the Fourier-Legendre series. Finally we provide conclusions and perspective on possible future developments and applications of this approach.

\section{POLARIMETRIC COHERENCE TOMOGRAPHY AlgORITHM}

\section{A. POLInSAR Height and Tomography Retrieval Algorithms}

The complex interferometric coherence observed for a vertical distribution of scatterers can be formulated as $[2,8]$

$$
\tilde{\gamma}=e^{i \phi_{0}} \frac{\int_{0}^{h_{v}} f(z) e^{i k_{z} z} d z}{\int_{0}^{h_{v}} f(z) d z}
$$

where $\phi_{0}=k_{z} z_{0}$ is the ground topography phase, $z_{0}$ is the position of the bottom of the vegetation layer, and $f(z)$ is the vertical structure function. In the special case of a 2-layer model, as used in POIInSAR for example [1, 2], the coherence can be rewritten as

$$
\tilde{\gamma}(\underline{w})=e^{i \phi_{0}} \frac{\tilde{\gamma}_{v}\left(h_{v}, \sigma\right)+\mu(\underline{w})}{1+\mu(\underline{w})}
$$

where $h_{v}$ - the height of volume, $\sigma$ - the mean extinction coefficient, $\mu$ - the ratio of surface-to-volume scattering and $\underline{\mathrm{w}}$ the polarization state. This relation can be used to find estimates of $h_{v}$ and $\phi_{0}$ in (1) as follows. We consider a dual polarization channel measurement system, $\underline{w}_{\mathrm{v}}$ (which we assume contains only volume scattering i.e. $\mu=0$ ) and the 
second $\underline{w}_{s}$ which contains an unknown mixture of surface and volume. The ground topography phase can then be found indirectly by using the coherences in the two channels as shown in (3) [7]

$$
\left.\begin{array}{l}
\tilde{\gamma}_{w_{v}}=e^{i \phi_{0}} \tilde{\gamma}_{v} \\
\tilde{\gamma}_{w_{s}}=\frac{e^{i \phi_{0}}\left(\tilde{\gamma}_{v}+\mu_{2}\right)}{1+\mu_{2}}
\end{array}\right\} \Rightarrow e^{i \phi_{0}}=\frac{\widetilde{\gamma}_{w_{v}}-\widetilde{\gamma}_{w_{s}}\left(1-L_{w_{s}}\right)}{L_{w_{s}}}
$$

The phase can be obtained by solving for $L_{w_{s}}$, itself obtained as the appropriate root of a quadratic equation as shown in (4)

$$
\begin{gathered}
\hat{\phi}=\arg \left(\widetilde{\gamma}_{w_{v}}-\widetilde{\gamma}_{w_{s}}\left(1-L_{w_{s}}\right)\right) 0 \leq L_{w_{s}} \leq 1 \\
A L_{w_{s}}^{2}+B L_{w_{s}}+C=0 \Rightarrow L_{w_{s}}=\frac{-B-\sqrt{B^{2}-4 A C}}{2 A} \\
A=\left|\widetilde{\gamma}_{w_{v}}\right|^{2}-1, B=2 \operatorname{Re}\left(\left(\widetilde{\gamma}_{w_{v}}-\widetilde{\gamma}_{w_{s}}\right) \cdot \widetilde{\gamma}_{w_{s}}^{*}\right), C=\left|\widetilde{\gamma}_{w_{v}}-\widetilde{\gamma}_{w_{s}}\right|^{2}
\end{gathered}
$$

To estimate of height $h_{v}$, we propose a new algorithm as shown in equation 5 . The main objective is to devise an algorithm which is robust to vertical structure variations. To achieve this, the height estimate comprises two components. The first is a phase difference between the volume channel and ground phase estimate from (4). As mentioned in [7], this by itself generally underestimates height and so needs augmenting by the second term, which is based on inversion of the coherence amplitude. Hence $h_{v}$ is given by

$$
h_{v}=\frac{\arg \left(\widetilde{\gamma}_{w_{v}}\right)-\hat{\phi}}{k_{z}}+\varepsilon \frac{\left.2 \sin c^{-1}\left(\tilde{\gamma}_{w_{v}}\right)\right)}{k_{z}}
$$

According to [7,9], choosing $\varepsilon=0.4$ leads to robust height estimation in varying forest density and structure environments.

\section{A. Fourier-Legendre Series Expansion and Vertical Structure Function}

We consider techniques for the reconstruction of $\mathrm{f}(\mathrm{z})$, the vertical structure function itself. From (1), we first remove the topography phase term and normalize the range of the integral by a change of variable as shown by

$$
\int_{0}^{h_{v}} f(z) e^{i k z} d z \stackrel{z^{\prime}=\frac{2 z}{h_{v}}-1}{\longrightarrow} \int_{-1}^{1} f\left(z^{\prime}\right) e^{i k z^{\prime}} d z^{\prime}
$$

We also rescale variation of the real non-negative function $\mathrm{f}(\mathrm{z})$ so that if $0 \leq f(z) \leq \infty$ then $f\left(z^{\prime}\right)=f(z)-1$ and $-1 \leq f\left(z^{\prime}\right) \leq \infty$. Therefore $\mathrm{f}\left(\mathrm{z}^{\prime}\right)$ can be developed as a Fourier-Legendre series on $[-1,1]$ as shown in (7)

$$
f\left(z^{\prime}\right)=\sum_{n} a_{n} P_{n}\left(z^{\prime}\right) \quad a_{n}=\frac{2 n+1}{2} \int_{-1}^{1} f\left(z^{\prime}\right) P_{n}\left(z^{\prime}\right) d z^{\prime}
$$

Therefore the coherence in (1) can be rewritten as

$$
\begin{aligned}
& \tilde{\gamma}_{v}=e^{i \frac{k_{z} h_{v}}{2} \int_{-1}^{1}\left(1+f\left(z^{\prime}\right)\right) e^{i \frac{k_{z} h_{v}}{2} z^{\prime}} d z^{\prime}} \\
& \int_{-1}^{1}\left(1+f\left(z^{\prime}\right)\right) d z^{\prime} \\
& =e^{i k_{v}} \frac{\int_{-1}^{1}\left(1+\sum_{n} a_{n} P_{n}\left(z^{\prime}\right)\right) e^{i k_{v} z^{\prime}} d z^{\prime}}{\int_{-1}^{1}\left(1+\sum_{n} a_{n} P_{n}\left(z^{\prime}\right)\right) d z^{\prime}} \\
& =e^{i k_{v}} \frac{\left(1+a_{0}\right) f_{0}+a_{1} f_{1}+a_{2} f_{2}+. .+a_{n} f_{n}}{\left(1+a_{0}\right)}
\end{aligned}
$$

where $k_{v}=\frac{k_{z} h_{v}}{2}$ and the first few functions $f_{i}$ are given as [7]

$$
\begin{aligned}
& f_{0}=\frac{\sin k_{v}}{k_{v}} \\
& f_{1}=i \frac{\sin k_{v}}{k_{v}^{2}}-\frac{\cos k_{v}}{k_{v}} \\
& f_{2}=\frac{3 \cos k_{v}}{k_{v}^{2}}-\left(\frac{6-3 k_{v}^{2}}{2 k_{v}^{3}}+\frac{1}{2 k_{v}}\right) \sin k_{v}
\end{aligned}
$$

\section{B. Single Baseline Coherence Tomography}

We can now rewrite the coherence for an arbitrary vertical structure function $\mathrm{f}(\mathrm{z})$ as

$$
\tilde{\gamma} e^{-i k_{v}}=\widetilde{\gamma}_{k}=f_{0}+a_{10} f_{1}+a_{20} f_{2}+\ldots+a_{n 0} f_{n}
$$

where $a_{n 0}=\frac{a_{n}}{1+a_{0}}$. If the set of unknown coefficients $a_{n 0}$ can be estimated, then we can use them to generate an estimate of the unknown vertical structure profiles. Since such function is reconstructed from a set of projections, i.e. the coherence, we now can solve this problem as an example of tomography by inverting the coherence for a single baseline interferometer as follows. First we rotate the phase of the coherence as shown in (11)

$$
\widetilde{\gamma}_{k}(w)=\widetilde{\gamma}(w) e^{-i k_{v}} e^{-i \hat{\phi}}
$$

and then we solve for the unknown Legendre coefficients by matrix inversion as shown in (12)

$$
\left[\begin{array}{l}
a_{00} \\
a_{01} \\
a_{02}
\end{array}\right]=\left[\begin{array}{ccc}
1 & 0 & 0 \\
0 & f_{1 i} & 0 \\
0 & 0 & f_{2}
\end{array}\right]^{-1}\left[\begin{array}{c}
1 \\
\operatorname{Im}\left(\widetilde{\gamma}_{k}\right) \\
\operatorname{Re}\left(\widetilde{\gamma}_{k}\right)-f_{0}
\end{array}\right]
$$

Finally we can estimate the vertical structure from

$$
\hat{f}(z)=a_{00}+a_{01} z+\frac{a_{02}}{2}\left(3 z^{2}-1\right)
$$


where $0 \leq z_{h} \leq h_{v} \Rightarrow-1 \leq z=\frac{2 z_{h}}{h_{v}}-1 \leq 1$.

\section{Single Baseline PCT AND GB-POLInSAR Data}

In [9] we investigated the ability of single baseline POLInSAR to retrieve vegetation height for short native Australian plants. Here we extend the analysis to investigate the ability of PCT to estimate variations in vertical profile due to density variations in the medium.

\section{A. Simulation of GB-POLInSAR Plants Measurement}

The sample consists of a group of short plants with a maximum height of $0.45 \mathrm{~m}$ planted in a round container of diameter $0.5 \mathrm{~m}$. They are evergreen plants with dense glossy green leaves. The vegetation sample is placed on a rotating turntable so that measurements can be made over 360 degrees of azimuth for a given angle of incidence. Wide-band POLInSAR data were acquired across the frequency range 3 to $6 \mathrm{GHz}$ at 70.8 degrees of the incident angle with a baseline of $10 \mathrm{~cm}$, as detailed in [9]. Note finally that the focus for the sensor (zero phase position for interferometry) is located around $2 \mathrm{~cm}$ lower than the ground surface of the sample which is $40 \mathrm{~cm}$ above the floor level of the chamber. Hence the ground phase parameter $\phi$ will not be zero and will change with frequency and baseline. We use two channels, cross polarized (HV) and the mixed surface-and-volume channel (HH-VV) for comparison. Therefore $\mathrm{HV}$ and $\mathrm{HH}-\mathrm{VV}$ coherences for measurement data can be obtained according to [9].

We use these parameters to simulate volume scattering from such a medium. The simulation was carried out with a $0.45 \mathrm{~m}$ high volume at 71 degrees angle of incidence with varying extinction from 0 to $20 \mathrm{~dB} / \mathrm{m}$. The coherence is first calculated for such a medium and then the Legendre coefficients calculated from coherence using equations 11 and 12. The Legendre tomogram is shown in Fig. 1.

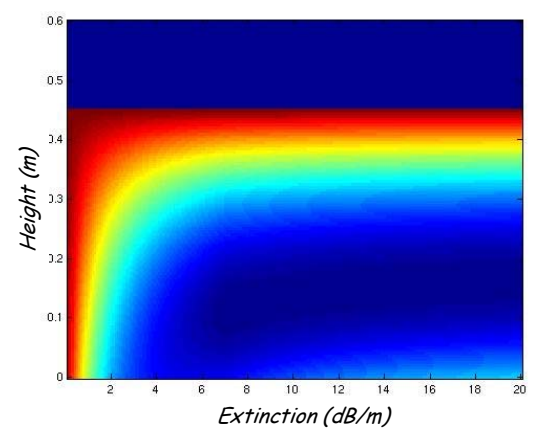

Figure 1. Simulated Legendre tomogram for extinction variation

Note that these tomograms do not depend on $\mathrm{k}_{\mathrm{z}}$ (baseline) and for zero extinction there is full volume scattering while for high extinction the dominant scattering comes from the upper layer. The Legendre vector is $(1, \mathrm{~L} 1, \mathrm{~L} 2)$ from equation 12 , where L1 is the linear coefficient and L2 the quadratic. The pairs L1, L2 for this simulation are shown in red in Figs. 2 and
3, which also show the experimental results for $\mathrm{HV}$ and $\mathrm{HH}-$ VV channels respectively.

We can see the variations of Legendre pair (L1, L2) with frequency. In red is the extinction model showing a steady increase in L1 and L2. The blue curve is the experiment data, showing departure from simple extinction model at high frequencies. This means that there is more to this structure than a simple extinction term (e.g. some other functional variation showing interesting structure to be investigated).

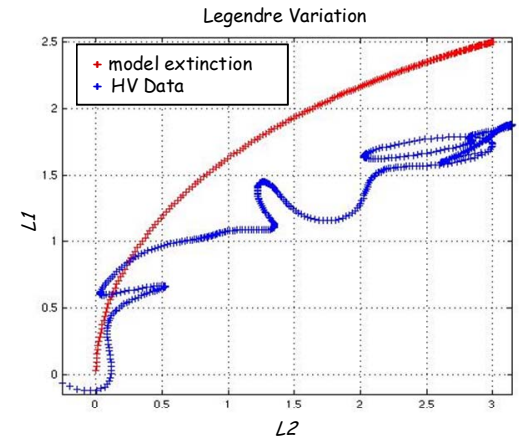

Figure 2. Legendre coefficients L1, L2 vs. frequency: HV channel

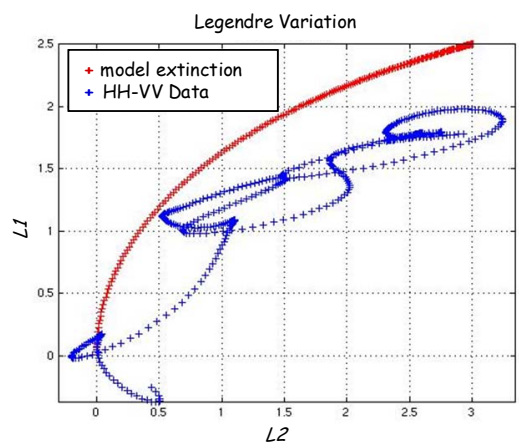

Figure 3. Legendre coefficients L1, L2 vs. frequency: HH-VV channel

\section{B. Tomographic Reconstructions using Known Height and Topography}

In our chamber measurement we have a priori knowledge from the geometry, of the ground phase and vegetation height in equation 1 and so do not need to employ equation 4 and 5 . However we can use the data to verify the suitability of HV and $\mathrm{HH}-\mathrm{VV}$ for height estimation based on the new algorithm in equation 5. Based on the true height of plants, the ground phase and measured coherences, tomograms for the plants can be reconstructed by equations 11 and 12. Fig. 4 shows the variation of vertical tomogram with frequency for the HV channel. We find that at low frequencies there is full volume scattering while at high frequency we see extinction structure which is consistent with the simulation in Fig. 1. The same phenomena are observed in Fig. 5 for the HH-VV channel.

Furthermore, variations of three different coherences with frequency are shown in Fig. 6 for the HV and HH-VV channels. In red is the original coherence from the measurement. In green is the phase corrected coherence (from equation 11). In blue is the 'Sinc' shifted coherence (equation 12). The main point is the blue curve, which starts at the origin 
(which corresponds to a uniform volume) and then moves as extinction increases with frequency. There are only slight differences for HH-VV channel.

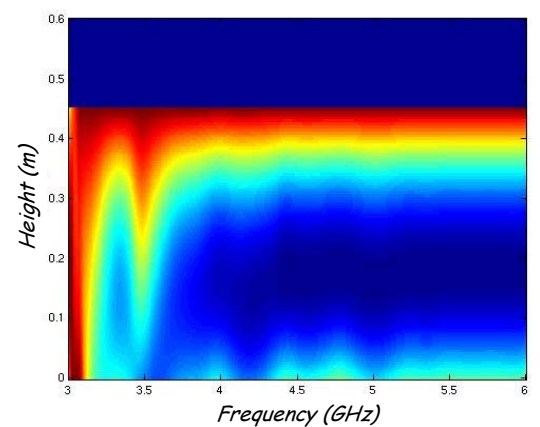

Figure 4. Polarimetric tomographic reconstruction of HV using the true height and ground topography

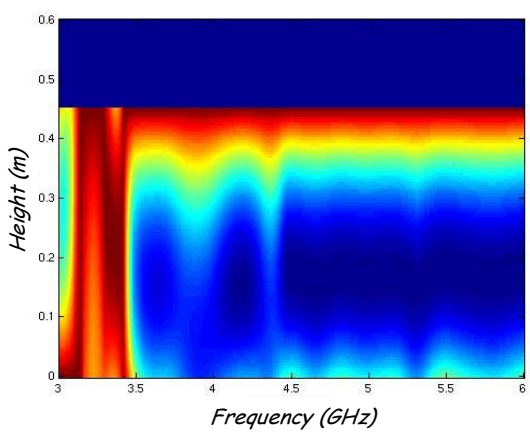

Figure 5. Polarimetric tomographic reconstruction of $\mathrm{HH}-\mathrm{VV}$ using the true height and ground topography

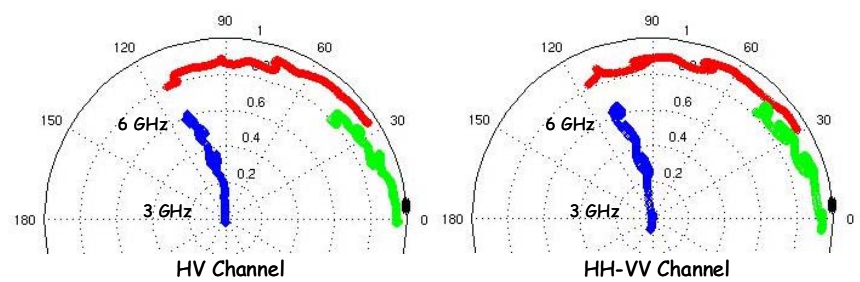

Figure 6. Coherence loci vs. frequency: red-measured, green-phase corrected, blue-Sinc shifted coherences

These results at 71 degrees angle of incidence show no significant polarization differences between $\mathrm{HH}-\mathrm{VV}$ and $\mathrm{HV}$ channels. Hence we conclude as follows: the short native vegetation is acting as a structured random volume, where there is no polarization dependence but demonstrable structure, it being measurably different from either a simple uniform volume or single extinction process. Therefore we propose use of $\mathrm{L} 1 / \mathrm{L} 2$ to classify different vegetation types in future applications.

\section{CONCLUSIONS}

In this paper we have reviewed a new radar imaging technique, Polarization Coherence Tomography (PCT). The method extends conventional polarimetric interferometry by allowing reconstruction of a vertical profile function, physically representing the variation of backscatter as a function of height. The approach depends on two key structure parameters of the vegetation under investigation, namely top height and ground phase. These two parameters can be obtained by a variety of methods, such as field measurements, other sensors (such as Lidar) or can themselves be estimated from the radar data in advance of application of PCT by using standard POLInSAR algorithms. With these parameters in place, the tomographic reconstruction problem can then be reformulated as a Fourier-Legendre series expansion and the unknown expansion coefficients estimated from coherence data by a single baseline, dual polarized interferometer.

We demonstrated how such a technique provides advanced abilities to investigate vertical structure features in a groundbased POLInSAR environment. Future analysis is required to investigate the dual and multiple baseline situation in more detail. Finally, although we have focused on application of PCT to vegetation mapping using ground-based radar imaging system, we believe this technique offers a wider range of application in any microwave scattering scenario where there is a combination of surface and volume scattering effects, such as improved vegetation species classification, improved biomass estimation and sub-canopy surface parameter mapping.

\section{ACKNOWLEDGMENT}

We would like to thank Professor B. D. Bates of Adelaide University for his support and constructive comments on this paper.

\section{REFERENCES}

[1] S. R. Cloude, K. P. Papathanassiou, "Polarimetric SAR interferometry," IEEE Trans. Geosci. Remote Sensing, vol. 36, pp. 1551-1565, September 1998

[2] K. P. Papathanassiou, S. R. Cloude, "Single baseline polarimetric SAR interferometry," IEEE Trans. Geosci. Remote Sensing, vol. 39, pp. 2352-2363, November 2001

[3] R.N. Treuhaft, G.P.Asner, B.E. Law, S. Van Tuyl, "Forest leaf area density profiles from the quantitative fusion of radar and hyperspectral data," J. Geophys. Res., vol. 107, no. D21, 4568, November 2002.

[4] A. Reigber, A. Moreira, "First demonstration of airborne SAR tomography using multi-baseline L band data," IEEE Trans. Geosci. Remote Sensing, vol. 38, no. 5, pp. 2142-2152, September 2000.

[5] R.N. Treuhaft, P. Siqueria,"Vertical structure of vegetated land surfaces from interferometric and polarimetric radar," Radio Science, vol. 35(1), pp. 141-177, January 2000.

[6] F. Lombardini, "Differential tomography: a new framework for SAR interferometry," IEEE Trans. Geosci. Remote Sensing, vol. 43, pp. 3744, January 2005.

[7] S. R. Cloude, "Polarisation coherence tomography," Radio Science, in press.

[8] S. R. Cloude, K. P. Papathanassiou, "A 3-stage inversion process for polarimetric SAR interferometry," IEE Proc. Radar Sonar Navigation, vol. 150, no. 3, pp. 125-134, June 2003.

[9] Z.-S. Zhou, S. R. Cloude, "Structural parameter estimation of australian flora with a ground-based polarimetric radar interferometer," in Proc. of IGARSS 2006, Denver, July 2006. 